3. Sorlozano A, Gutierrez J, Roman E, de Dios LJ, Roman J, Liebana $\mathrm{J}$, et al. A comparison of the activity of tigecycline against multiresistant clinical isolates of Staphylococcus aureus and Streptococcus agalactiae. Diagn Microbiol Infect Dis. 2007;58:487-9.

4. Alvarez Lerma F, Blanco L, Rodriguez JA, Grau S, Conde Estevez $D$, Luque $S$. Diferencias en el uso de tigeciclina entre pacientes críticos y no críticos. Rev Esp Quimioter. 2010;23:63-71.

5. Swoboda S, Ober M, Hainer C, Lichtenstern C, Seiler C, Wendt $\mathrm{C}$, et al. Tigecycline for the treatment of patients with severe sepsis or septic shock: a drug use evaluation in a surgical intensive care unit. J Antimicrob Chemother. 2008;61:729-33.
E. Curiel Balsera*, A.M. Poullet Brea y

M.Á. Prieto Palomino

Unidad de Cuidados Intensivos, Hospital Regional

Universitario Carlos Haya, Málaga, España

* Autor para correspondencia.

Correo electrónico: emiliouci@telefonica.net

(E. Curiel Balsera).

doi:10.1016/j.medin.2010.09.003

\section{Hemorragia cerebelosa secundaria a fuga de líquido cefalorraquídeo tras cirugía del canal lumbar}

\section{Cerebellar hemorrhage secondary to cerebros- pinal fluid leak after lumbar canal surgery}

\section{Sr. Director:}

La hemorragia cerebelosa a distancia (HCD) se considera una complicación rara de la cirugía intracraneal y excepcional tras la intervención espinal ${ }^{1}$. Presentamos el caso de una mujer que desarrolla una HCD en el postoperatorio de estenosis de canal lumbar.

Se trata de una mujer de 58 años de edad, con antecedentes de síndrome depresivo, que es intervenida de estenosis de canal lumbar, realizándosele una laminectomía L5-S1 que se complica con un desgarro de la duramadre, con salida de líquido cefalorraquídeo (LCR), que obliga a su sutura. A las 36 horas de la cirugía, y estando en tratamiento con analgésicos mórficos y sedantes, presenta depresión del nivel de conciencia que revierte completamente con naloxona y flumazenil intravenosos. A las 12 horas de este episodio, y ya sin los fármacos previamente descritos, vuelve a presentar fluctuaciones de la conciencia y cefalea, por lo que se realiza un tomografía computarizada cerebral (TC) que muestra una pequeña hemorragia lineal y otra mayor $(1,4$ $x 0,9 \mathrm{~cm}$ ) en el hemisferio cerebeloso derecho e izquierdo respectivamente. Hemodinámicamente se mantiene estable y la bioquímica, hemograma y coagulación muestran valores normales. La paciente se traslada a un centro hospitalario con servicio de neurocirugía, en donde evoluciona satisfactoriamente con tratamiento conservador.

La HCD se ha descrito tras cirugía supratentorial, presentando una incidencia que varia desde el $0,2 \%$ hasta alcanzar el 3,5-4,9\%, en el caso de intervenciones de aneurismas y después de lobectomías temporales, siendo incluso menor y observándose ocasionalmente después de cirugía raquídea, aunque la tasa real probablemente sea superior al infradiagnosticarse los casos asintomáticos ${ }^{1-4}$. Aunque la etiopatogenia está sujeta a discusión, pudiendo estar involucradas alteraciones de la coagulación, hipertensión arterial y rotación de la cabeza dificultando el drenaje venoso cerebral, la mayoría de autores lo atribuyen a un síndrome de hipotensión de LCR, secundario a pérdida del mismo tras la lesión dural, que secundariamente produciría un descenso del cerebelo con tracción y obstrucción sobre las venas del puente y finalmente un infarto venoso hemorrágico ${ }^{1-4}$. Característicamente las hemorragias son bilaterales y se localizan preferentemente en el vermis y surcos cerebelosos, como en nuestro caso, revelando a veces la TC cerebral una imagen curvilínea hemorrágica en dichos surcos, conocido como el signo de la cebra ${ }^{4,5}$. Clínicamente cursan con cefalea, que no mejora claramente con el decúbito, alteraciones de la conciencia y a veces se acompañan de signos neurológicos focales. El pronóstico depende fundamentalmente de la edad y de la gravedad de la hemorragia, quedando sin o con discretas secuelas neurológicas más del $50 \%$ de casos, llegando a alcanzar una mortalidad del 10 $15 \%{ }^{1}$. El tratamiento varía desde una actitud conservadora hasta cirugía intracraneal urgente, debiendo comprobarse sin demora si existe fuga de LCR y en su caso proceder a reparar urgentemente la fuga dural.

Concluimos haciendo hincapié en que la presentación de cefalea acompañada a veces de fluctuaciones del nivel de conciencia, con o sin disfunción cerebelosa o de tronco, en el postoperatorio de cirugía raquídea, obliga a realizar urgentemente una TC craneal para descartar una HCD.

\section{Bibliografía}

1. Brockmann MA, Groden C. Remote cerebellar hemorrhage: a review. Cerebellum. 2006;5:64-8.

2. Riera Sagrera M, Bergadá García J. Hemorragia cerebelosa tras cirugía espinal. Med Intensiva. 2008;32:54-5.

3. Karaeminogullari O, Atalay B, Sahin O, Ozalay M, Demirors H, Tuncay $C$. Remote cerebellar hemorrhage after a spinal surgery complicated by dural tear: Report and literatura review. Neurosurgery. 2005;57(Supl 1):E215.

4. Nam TK, Park SW, Min BK, Hwang SN. Remote cerebellar hemorrhage after lumbar spinal surgery. J Korean Neurosurg. 2009;46:501-4.

5. Brockmann MA, Nowak G, Reusche E, Russlies M, Petersen D. Zebra sign: cerebellar bleeding pattern characteristic of cerebrospinal fluid loss. J Neurosurg. 2005;102:1159-62.

M.A. Blasco Navalpotro*, F. Del Nogal Sáez y R. Díaz Abad

Servicio de Medicina Intensiva, Hospital Universitario Severo Ochoa. Leganés, Madrid, España

* Autor para correspondencia. Correo electrónico: mblasco.hsvo@salud.madrid.org (M.A. Blasco Navalpotro).

doi:10.1016/j.medin.2010.09.008 